Review Article

\section{Chronic fatigue syndrome and epigenetics: The case for hyperbaric oxygen therapy in biomarker identification}

\author{
Rajit J Shah*
}

Duke University, Sugar Land, Texas, United States

\section{Abstract}

Chronic fatigue syndrome (CFS) is a poorly-understood respiratory condition that affects millions of individuals. Hyperbaric oxygen therapy (HBOT) is a treatment option being considered to address CFS as it is suggested to combat fatigue and increase oxygenation. HBOT provides two opportunities in advancing research of CFS: it may provide data on symptom amelioration and be utilized in the search for a biomarker. By either identifying biomarkers before using HBOT to compare epigenomes of patients before and after treatment or using HBOT to find epigenetic discrepancies between patients with and without treatment, matching epigenetic regulation with symptom amelioration may significantly advance the understanding of the etiology and treatment mechanism for CFS. EPAS1/HIF-2 $\alpha$ is a leading candidate for an epigenetic biomarker as it responds differentially to hypoxic and normoxic conditions, which degrades more slowly in hypoxic conditions. Epigenetic regulation of EPAS1/HIF-2 $\alpha$ in such differential conditions may be explored in HBOT experiments. In addition to HBOT as a promising treatment option for CFS symptoms, it may aid the identification of biomarkers in CFS. Further research into both outcomes is strongly encouraged.

\section{More Information}

*Address for Correspondence: Rajit J Shah, Duke University, Sugar Land, Texas, USA, Email: rajit.shah@duke.edu

Submitted: January 06, 2021

Approved: February 25, 2021

Published: February 26, 2021

How to cite this article: Shah RJ. Chronic fatigue syndrome and epigenetics: The case for hyperbaric oxygen therapy in biomarker identification. J Pulmonol Respir Res. 2021; 5: 027-030.

DOI: 10.29328/journal.jprr.1001020

Copyright: @ 2021 Shah RJ. This is an open access article distributed under the Creative Commons Attribution License, which permits unrestricted use, distribution, and reproduction in any medium, provided the original work is properly cited.

Keywords: Chronic fatigue syndrome; Hyperbaric oxygen therapy; EPAS1; HIF-2a; Hypoxia; Epigenetic regulation

(D) Check for updates

OPEN ACCESS

\section{Introduction}

Chronic fatigue syndrome, also known as myalgic encephalomyelitis or ME/CFS, is a respiratory disease that affects more than 4 million Americans [1]. CFS is diagnosed when fatigue is systematically observed for at least six months [2]. Despite such guidelines for diagnosis, the etiology of CFS remains unknown, suggesting that a significant amount of research is required to develop effective treatment options $[3,4]$. The condition hinders individuals from engaging in regular physical activity compared to those without CFS with severe fatigue and headaches following exercise [5]. The scope and magnitude of the disease warrants investigation of both the etiological mechanism for CFS onset as well as a survey of potential treatment options. One potential treatment under review is hyperbaric oxygen therapy (HBOT), which delivers oxygen at pressures up to three times higher than normal air pressure, increasing oxygenation of the lungs to promote healing [6]. HBOT is a candidate treatment in various conditions, such as decompression sickness and wounds from radiation injury [6]. However, research into the effects of HBOT on CFS is limited, particularly an understanding of how it may alleviate symptoms or its potential impact on the epigenome. Therefore, in tandem with epigenetic analysis to find CFS-associated biomarkers, a nuanced approach towards evaluating patient outcomes of HBOT on symptom amelioration as well as epigenome modifications must be conducted to advance efforts to better understand the etiology and treatment mechanisms for CFS.

\section{Neuropathic comorbidity}

The effects of CFS may be accumulated over time plausibly because a lack of exercise can result in other conditions induced by inactivity. It is understood that exercise and activity is beneficial for overall health, but there are significant implications pertaining to the epigenome and neuropathology. Exercise has been linked to an increased expression of brain-derived neurotrophic factor (BDNF), which is associated with an improvement in cognitive function and a reduction in depression and anxiety [7]. This occurs through D- $\beta$-hydroxybutyrate inhibiting HDAC2 and HDAC3, which are BDNF inhibitors, causing an increase in neurotransmitter release at the CA3-CA1 synapse in the hippocampus. Such activation is involved with a vast slate of other regulatory downstream mechanisms that are believed 
to improve memory and cognition [7]. Such a pathway provides a glimpse of the intricate biological machinery required to maintain normal functioning, suggesting that a lack of exercise caused by CFS may disrupt neuroregulation (Figure 1). In the context of CFS, patients were seen to reflect a reduced overall white matter among other abnormalities involving gray matter thickness and structure of the right arcuate fasciculus [8]. These findings contribute to beliefs that CFS may play a role in the propensity of patients developing neuropathic comorbidities. In general, the ramifications of CFS on the central nervous system comprise just one category of complications associated with CFS.

\section{Rationale for HBOT in biomarker identification}

Respiratory biomarker identification studies are not new. Tests observing pollution on rat models discovered a link between traffic-related air pollution (TRAP) and an increase in epigenetic marks found on histone proteins [9]. These tests suggest a reverse causality; if pollution leaves an epigenetic mark, the absence of pollution should also leave its own 'mark' by the absence of an anticipated biomarker.

The same line of reasoning may apply in finding biomarkers for CFS. Studies have indicated a connection between epigenetic regulation and CFS, however, there are difficulties in finding biomarkers directly responsible for changes in fatigue [2]. Thus, further research is strongly recommended, leaving room for HBOT to advance CFS literature. Comparing the relevant segments in the epigenomes of patients with and without CFS could reveal regions of interest (ROIs) for further research. Such a subtractive approach may be an effective tool in order to identify the specific locations of CFS biomarkers. Once ROIs are identified, HBOT studies may estimate efficacy of treatment at the physiological level where patients could

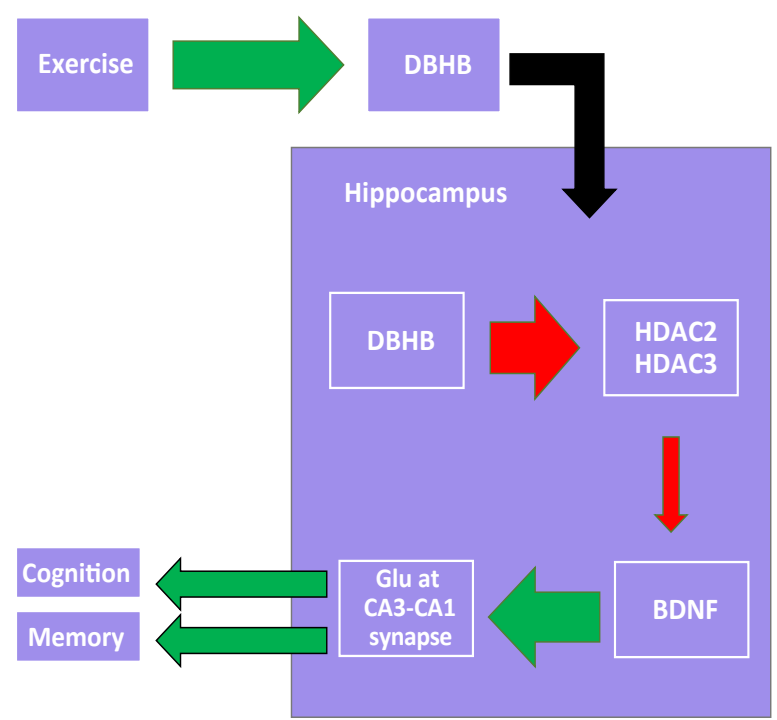

Figure 1: A proposed model by which exercise enhances cognition and memory. Upregulation of DBHB synthesis enhances the inhibitory effect on HDAC2 and HDAC3, which inhibit hippocampal BDNF expression. BDNF upregulation enhances glutamate release from CA3 onto CA1 in the trisynaptic circuit. Glutamate release is linked to improvements in cognition and memory. report symptom amelioration and at the epigenetic level where symptom analysis could show activation or inactivation of involved sites and confirm epigenome-physiology interplay. Another approach to identifying biomarkers would be to directly compare epigenetic regulation in CFS patients before and after HBOT along with a control cohort of healthy individuals. In summary, experiments exploring comparative epigenome analysis in CFS and healthy patients along with HBOT outcome analysis may reveal crucial developments in understanding the etiology and treatment mechanisms in CFS.

\section{Preliminary research of HBOT in CFS symptom amelio- ration}

Hyperbaric oxygen therapy (HBOT) exposes the body to $100 \%$ oxygen at an altitude pressure higher than $1 \mathrm{~atm}$ and may occur through an oxygen mask for lung treatment, or in the hyperbaric chamber for a full body treatment $[6,10,11]$ According to our knowledge, there have very few been attempts to observe the impact of HBOT on CFS, which have not been conclusive. Despite such limitations, some progress has been made regarding symptom amelioration of CFS and HBOT. At the symptomatic level, HBOT was associated with slight improvements in humans on the Fatigue Severity Scale (FSS), which is a 9-63 point scaled questionnaire containing nine statements related to the impacts of fatigue on patient routines - e.g. how fatigue symptoms affect exercise and social life; the results concluded a statistically significant $(p<0.005)$ reduction in fatigue severity from 53.20 to 14.60 [11]. This is the only clinical, human trial that directly observes the potential of HBOT in symptom amelioration to our knowledge. The limitation of such a study is the rather limited sample size $(n=16)$ along with the lack of a control group, female participants, and a follow-up trial. Therefore, more thorough trials following this preliminary one are strongly suggested. One study found that HBOT on CFS patients slightly decreased fatigue, but didn't establish the desired impact on increasing activity, which slightly decreased instead [12]. These results suggest the potential of HBOT as at least an adjunct treatment option. However, further research with larger sample sizing is required to advance these early findings.

\section{Preliminary research of oxygenation in biomarker iden- tification}

Although research has been conducted on potential biomarkers and the effects of HBOT, only a few of them take into account epigenetic regulation [2,11,12]. One epigenetic approach examines the genes associated with oxygen leveling. The EPAS1/HIF-2 $\alpha$ gene plays a role in adjusting the body to changing oxygen levels $[13,14]$. EPAS1 is located at $2 \mathrm{p} 21$ : chromosome 2, arm p, position 21 [13]. It is responsible for the production of a protein called hypoxia-inducible factor 2-alpha (HIF-2 $\alpha$ ) [13]. The HIF complex regulates the formation of blood vessels, the production of red blood cells, 
and hemoglobin concentration to maximize the amount of oxygen in the body $[13,15]$. EPAS1 is involved with the body's response to higher altitude, the exact conditions HBOT provides $[13,15]$.

While coverage of the mechanism for EPAS1 and the HIF complex is currently limited, recent research has improved confidence in preliminary proposed mechanisms (Figure 2). The prevailing mechanism describes post-translational inhibitory regulation of HIF transcription under normoxic conditions. Hypoxic conditions have the reverse effect on HIF transcription, suggesting that oxygen levels have a scalar, observable impact on EPAS1 regulation [16]. Under hypoxic conditions, HIF heterodimers enter the nucleus and initiate oxygen transport via hypoxia-response elements (HREs) to hypoxic regions [16,17]. To carry oxygen to such regions, HREs may also stimulate erythropoiesis via increased erythropoietin (EPO) transcription, which in turn increases hemoglobin synthesis [18-21].

Mutation studies confirm multiple aspects of the proposed EPAS1/HIF- $2 \alpha$ pathway. Tumoral EPAS1/HIF- $2 \alpha$ mutations have been found in patients with congenital erythrocytosis, suggesting that erythropoiesis is at least partially regulated by a pathway involving EPAS1/HIF-2 $\alpha$ [22]. Epigenetic studies also contribute to the understanding of the pathway. Generally, differences in EPAS1/HIF- $2 \alpha$ expression can be observed in ducks and even humans living in relatively higher altitudes, suggesting that oxygen levels may have a scalar, differential impact on the pathway [16].

HIF- $2 \alpha$ is a promising candidate for an epigenetic

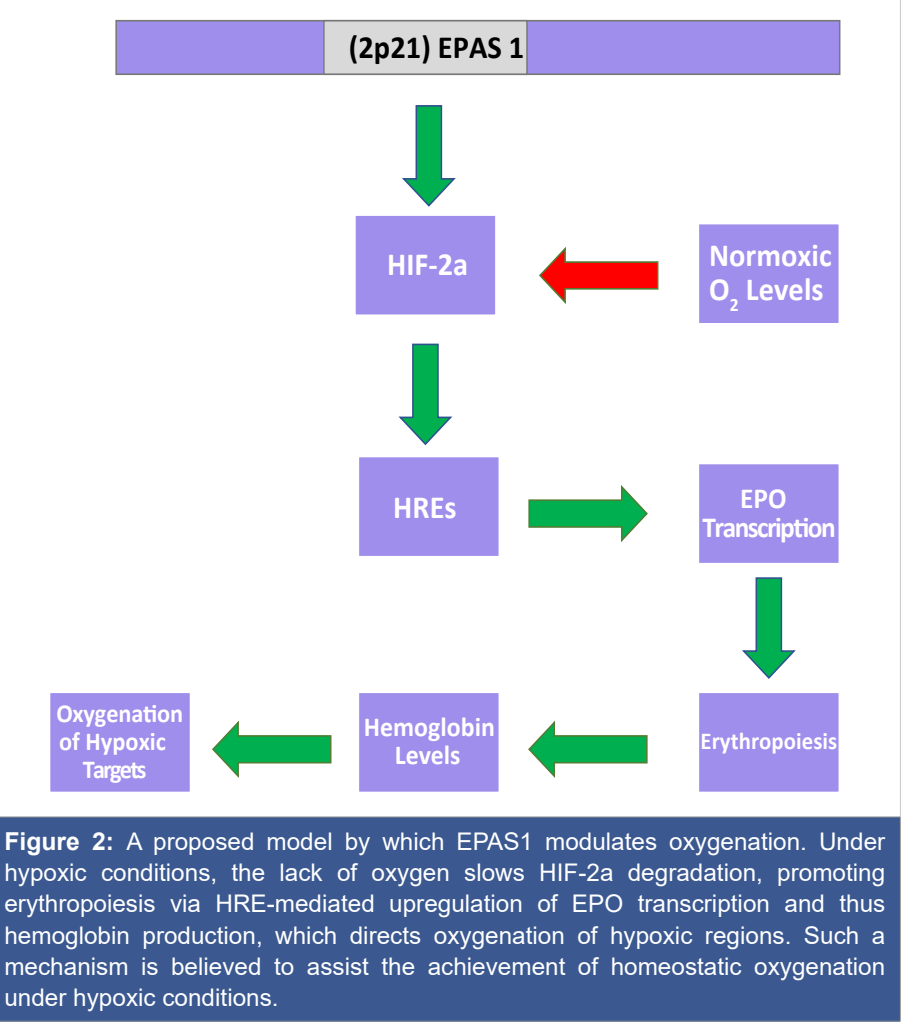

biomarker. When adequate oxygen is available, proteins should target HIF- $2 \alpha$ to be broken down [13]. When oxygen levels are lower than normal, however, HIF- $2 \alpha$ should be degraded at a slower rate [13]. This mechanism ties in with CFS as it is correlated with a decrease in oxygen levels, especially seen during exercise because a decreased amount of red blood cells indicates a reduced oxygen carrying power, consequently reducing oxygen-carrying power $[13,23]$. With these findings, CFS patients should present slower HIF- $2 \alpha$ degradation. Thus, one leading approach to identifying an epigenetic biomarker involved with CSF is the observation of the levels of HIF- $2 \alpha$ at 2 p21.

\section{Conclusion}

CFS requires significant amounts of further study. There is a lack of sufficient evidence to confidently establish any systematic approach to treating CFS [2]. Biomarkers have been researched, but thus far, the sample size is not enough to validate preliminary findings. There is neither a consensus of an established treatment method, nor a comprehensive analysis on the efficacy of HBOT on CFS. Preliminary research may provide leads into future studies. Promising studies covering the symptom-ameliorating effects of HBOT as well as variable EPAS1/HIF-2 $\alpha$ expression based on oxygenation must be further explored to potentially identify an epigenetic approach towards understanding the etiology and treatment mechanisms of CFS. Comparing epigenetic regulation before and after HBOT in patients with CFS could result in critical developments in addressing the disease.

\section{Acknowledgement}

I would like to acknowledge Amy Tsurumi, $\mathrm{PhD}$ of Harvard Medical School. I have no conflict of interest to declare.

\section{References}

1. Broderick G, Fuite J, Kreitz A, Vernon SD, Klimas N, et al. A formal analysis of cytokine networks in chronic fatigue syndrome. Brain Behav Immun. 2010; 24: 1209-1217.

PubMed: https://pubmed.ncbi.nlm.nih.gov/20447453/

2. Landmark-Høyvik $\mathrm{H}$, Reinertsen $\mathrm{KV}$, Loge JH, Kristensen VN, Dumeaux V, et al. The genetics and epigenetics of fatigue. PMR. 2010; 2: 456-465.

PubMed: https://pubmed.ncbi.nlm.nih.gov/20656628/

3. Friedberg F, Jason LA. Understanding chronic fatigue syndrome: An empirical guide to assessment and treatment. American Psychological Association. 1998.

4. Lombardi VC, Ruscetti FW, Gupta JD, Pfost MA, Hagen KS, et al. Detection of an infectious retrovirus, XMRV, in blood cells of patients with chronic fatigue syndrome. Science. 2009; 326: 585-589.

PubMed: https://pubmed.ncbi.nlm.nih.gov/19815723/

5. Jason LA, Porter N, Brown M, Anderson V, Brown A, et al. CFS: a review of epidemiology and natural history studies. Bull IACFS ME. 2009; 17: 88-106. PubMed: https://pubmed.ncbi.nlm.nih.gov/21243091/

6. Mayo Clinic. Hyperbaric oxygen therapy. https://www.mayoclinic.org/ tests-procedures/hyperbaric-oxygen-therapy/about/pac-20394380

7. Sleiman SF, Henry J, Al-Haddad R, El Hayek L, Abou Haidar E, et al. Exercise promotes the expression of brain derived neurotrophic factor 
(BDNF) through the action of the ketone body $\beta$-hydroxybutyrate. Elife. 2016; 5: e15092.

PubMed: https://pubmed.ncbi.nlm.nih.gov/27253067/

8. Goldman B. Some headway on chronic fatigue syndrome: Brain abnormalities pinpointed. 2014

http://scopeblog.stanford.edu/2014/10/29/some-headway-on-chronicfatigue-syndrome-brain-abnormalities-pinpointed/.

9. Ding R, Jin Y, Liu X, Zhu Z, Zhang Y, et al. H3K9 acetylation change patterns in rats after exposure to traffic-related air pollution. Environ Toxicol Pharmacol. 2016; 42: 170-175.

PubMed: https://pubmed.ncbi.nlm.nih.gov/26855416/

10. John's Hopkins Medicine. Hyperbaric Oxygen Therapy for Wound Healing.http://www.hopkinsmedicine.org/healthlibrary/test_procedures/ neurological/hyperbaric_oxygen_therapy_for_wound_healing_135,44/

11. Akarsu S, Tekin L, Ay $H$, Carli AB, Tok $F$, et al. The efficacy of hyperbaric oxygen therapy in the management of chronic fatigue syndrome. Undersea Hyperb Med. 2013; 40: 197-200. PubMed: https://pubmed.ncbi.nlm.nih.gov/23682549/

12. Hoof EV, Coomans D, Becker PD, Meeusen R, Cluydts R, et al Hyperbaric therapy in chronic fatigue syndrome. J Chronic Fatigue Syndr. 2003; 11: 37-49.

13. MedlinePlus. EPAS1 Gene. https://ghr.nlm.nih.gov/gene/EPAS$1 \#$ conditions

14. Henderson J, Withford-Cave JM, Duffy DL, Cole SJ, Sawyer NA, et al. The EPAS1 gene influences the aerobic-anaerobic contribution in elite endurance athletes. Hum Genet. 2005; 118: 416-423. PubMed: https://pubmed.ncbi.nlm.nih.gov/16208515/

15. Beall CM, Cavalleri GL, Deng L, Elston RC, Gao Y, et al. Natural selection on EPAS1 (HIF2 $\alpha$ ) associated with low hemoglobin concentration in ibetan highlanders. Proc Natl Acad Sci U S A. 2010; 107: 11459-11464. PubMed: https://pubmed.ncbi.nlm.nih.gov/20534544/

16. Graham AM, McCracken KG. Convergent evolution on the hypoxiainducible factor (HIF) pathway genes EGLN1 and EPAS1 in highaltitude ducks. Heredity. 2019; 122: 819-832.

PubMed: https://pubmed.ncbi.nlm.nih.gov/30631144/

17. Wenger RH, StiehI DP, Camenisch G. Integration of oxygen signaling at the consensus HRE. Sci STKE. 2005; 2005: re12.

PubMed: https://pubmed.ncbi.nlm.nih.gov/16234508/

18. Storti F, Santambrogio S, Crowther LM, Otto T, Abreu-Rodríguez I, et al. A novel distal upstream hypoxia response element regulating oxygen-dependent erythropoietin gene expression. Haematologica. 2014; 99: e45-48.

PubMed: https://pubmed.ncbi.nlm.nih.gov/24510339/

19. Lorenzo FR, Yang C, Fui MN, Vankayalapati H, Zhuang Z, et al. A novel EPAS1/HIF2A germline mutation in a congenital polycythemia with paraganglioma. J Mol Med. 2013; 91: 507-512.

PubMed: https://pubmed.ncbi.nlm.nih.gov/23090011/

20. Haase VH. Regulation of erythropoiesis by hypoxia-inducible factors. Blood Rev. 2013; 27: 41-53. PubMed: https://pubmed.ncbi.nIm.nih.gov/23291219/

21. Liu S, McConnell SC, Ryan TM. Erythropoiesis in the absence of adult hemoglobin. Mol Cell Biol. 2013; 33: 2241-2251. PubMed: https://pubmed.ncbi.nlm.nih.gov/23530053/

22. Comino-Méndez I, de Cubas AA, Bernal C, Álvarez-Escolá C, Sanchez-Malo C, et al. Tumoral EPAS1 (HIF2A) mutations explain sporadic pheochromocytoma and paraganglioma in the absence of erythrocytosis. Hum Mol Genet. 2013; 22: 2169-2176.

PubMed: https://pubmed.ncbi.nlm.nih.gov/23418310/

23. Streeten DH, BellMD DS. Circulating blood volume in chronic fatigue syndrome. J Chronic Fatigue Syndrome. 1998; 4: 3-11. 